\title{
Corrigendum: Complementary activities of TPX2 and chTOG constitute an efficient importin-regulated microtubule nucleation module
}

\section{Johanna Roostalu, Nicholas I. Cade and Thomas Surrey}

Nat. Cel/ Biol. http://dx.doi.org/10.1038/ncb3241 (2015); published online 28 September 2015; corrected online 5 October 2015

In the version of this Article originally published online there was an incorrect citation in the methods section. This sentence should have read "GMPCPP-stabilized biotinylated fluorescently labelled microtubule 'seeds' for assays with dynamic microtubules were prepared as described previously ${ }^{41}$ (containing 12\% of either Atto647N- or Atto565-labelled tubulin)”. This error has been corrected. 